Vol. 18 (2009): 144-151.

\title{
Genetic stability of in vitro conserved germplasm of Humulus lupulus $\mathrm{L}$.
}

\author{
Elena L. Peredo ${ }^{1 *}$, Rosa Arroyo-García ${ }^{2}$, Barbara M. Reed ${ }^{3}$, M. Ángeles Revilla ${ }^{1}$ \\ ${ }^{1}$ Universidad de Oviedo, Department of Plant Physiology, Catedrático Rodrigo Uría s/n, 33071 Oviedo, Spain \\ ${ }^{2}$ Departamento de Biotecnología, Instituto Nacional de Investigación Agraria y Alimentaria, \\ Carretera de A Coruña, km 7.5, 28040 Madrid, Spain \\ ${ }^{3}$ United States Department of Agriculture, Agricultural Research Service, National Clonal \\ Germplasm Repository, Corvallis, OR 97333-2521, USA \\ *e-mail: elperedo.uo@uniovi.es
}

The genetic and epigenetic stability of hop accessions cryopreserved for one year or cold stored for three years was evaluated using several molecular markers (RAPD, AFLP, and MSAP). Clear, repetitive patterns were obtained among accessions and between control and treated samples. Although no genetic changes were detected among the control plants grown in the greenhouse and in vitro plants regenerated from slow-cooling cryopreserved shoot tips or cold stored in vitro shoots, MSAP analysis detected methylation changes in $36 \%$ of the loci. Nevertheless, only 2.6 to $9.8 \%$ of the detected changes could be ascribed to the conservation procedure and most of them seemed to be generated as a result of the in vitro introduction. Due to the number of accessions analysed (51) we can cautiously deduce that the genetic behaviour described in this work after cryopreservation or cold-storage protocols is common to most hop genotypes and these storage procedures are suitable for standard use. However, it is important to keep in mind the epigenetic changes produced, particularly during any in vitro processes.

Key-words: AFLP (Amplified Fragment Length Polymorphism), cold storage, cryopreservation, MSAP (Methylation Sensitive Amplified Polymorphism), RAPD (Random Amplified Polymorphic DNA), somaclonal variation. 
Vol. 18 (2009): 144-151.

\section{Introduction}

Humulus lupulus L. is a dioecious climbing perennial of the Cannabaceae family indigenous to Europe, Asia, and North America. The female inflorescences (cones) are widely used to preserve beer and to give it a characteristic aroma and flavour. The appearance of downy mildew in the northern hemisphere has increased the need to breed new resistant varieties. Breeding programmes continue to focus on increasing hop alpha acid content and aroma profiles. Several cultivars were developed by crossing traditional European cultivars with wild plants from America, with the aim at combining the aroma qualities of the European varieties and the high yielding capacity of the American hops (Moir 2000).

It is extremely important to develop effective methods to store the many cultivars, as well as breeding lines and diverse wild material needed for developing new cultivars. Currently the most useful techniques to store plant material are refrigerated cold storage and cryopreservation at ultra-low temperatures, usually in liquid nitrogen. It is important to keep in mind the risk of genetic and epigenetic instabilities caused by any storage methods. Several factors associated with in vitro culture procedures, such as the medium composition (Viterbo et al. 1994), may result in somatic variation in cold stored plants. During cryopreservation, exposing tissues to physical, chemical and physiological stresses may result in cryoinjury, which ultimately may have effects at genome level (Harding 2004). Several molecular marker techniques are available to analyze genetic stability in plants. Two commonly used techniques are Amplified Fragment Length Polymorphism (AFLP) (Vos et al. 1995) and Random Amplified Polymorphic DNA (RAPD) (Williams et al. 1990). Epigenetic changes due to the stress generated during in vitro culture could possibly generate altered phenotypes in the recovered plants. This epigenetic instability is implicated in the timing of the DNA replication, in determination of chromatin structure, in increasing mutation frequency; as a causal agent for some human diseases; and as a basis for epigenetic phe- nomena (Finnegan et al. 1998). Methylation Sensitive Amplified Polymorphism technique (MSAP) can be used to identify methylation changes in anonymous CCGG regions using the isoschizomeric HpaII/MspI restriction enzymes.

The aim of this study was to evaluate the genetic stability of several hop accessions, representative of those growing around the world, in order to assess the efficacy of standard cryopreservation and/ or cold storage protocols for the diverse cultivars and wild species in genebank collections.

\section{Material and methods}

\section{Plant material}

Hop accessions (51) representing wild hops, breeding lines, and cultivars supplied by the USDA, National Clonal Germplasm Repository (Corvallis, OR, USA) in February 2005 were analyzed. Each accession (cryopreserved or cold stored) included a control sample and at least two replicated sample treatments. Cold storage and cryopreservation were performed according Reed et al. (2003). Potted greenhouse-grown plants from the USDA core collection were used as control samples. Cold storage was applied for in vitro plants at $4{ }^{\circ} \mathrm{C}$ with a 12-h photoperiod $\left(10 \mu \mathrm{molm}^{-2} \mathrm{~s}^{-1}\right)$ for one year. For cryopreservation, in vitro shoot tips were dissected from 2-week cold-acclimated plantlets and kept in liquid nitrogen (LN) for 3 years and regrown in the same in vitro conditions as the parent shoots for 4 months.

\section{Molecular techniques}

Altogether 51 hop accessions were analysed, seven accessions were cryopreserved and 36 were kept under cold storage conditions. In addition, samples of four accessions were independently conserved under both treatments. For each accession one control plant and at least two controls were analysed. 
Peredo, E.L. et al. In vitro genetic stability of hops

In total 169 samples were analysed. The RAPD reactions were carried out according to Pillay and Kenny (1996) with slight modifications. Twelve annealing temperatures were tested for each primer and the most appropriate annealing temperature was selected to avoid repetition problems and to increase the number of easy-scored DNA fragments. In all the tested primers, the highest temperature which produced the most suitable band profile was selected (see Table 1 for list of primers and annealing temperatures). In the AFLP assay the genetic stability of five cold-stored and five cryopreserved accessions was tested. We consider that these 10 selected accessions (Table 2) are representative of the total variation within Humulus lupulus as they include wild hops, females, males, diploid and triploids. AFLP analysis was performed according to Cervera et al. (1998). The primers used for the selective amplification were Eco AGC, AGA, AAC, and Mse CAT, CTT. A standard silver staining protocol was used to reveal the bands.

Three hop accessions representative of commercially cultivated hops were selected for the evaluation of methylation stability (Table 3 ). The MSAP analysis was performed following the general steps according to Cervera et al. (2002). For the selective amplification fluorochrome-labelled primers were used, HpaII/MspI +AAC, +ACT, +ACG (Applied Biosystem, CA, USA) combined with EcoRI +ACT, and AAT. Samples were electrophoresed in an automatic sequencer ABI PRISM ${ }^{\circledR}$ 3100 Genetic Analyzer (Applied Biosystem, CA, USA). The loci were considered polymorphic when differences in the presence/absence of bands in the EcoRI/MspI and EcoRI/HpaII patterns among the control and the treated samples were detected.

\section{Results}

Eleven RAPD primers were selected for the assay. A total of 125 loci were detected with a mean of 11 loci per primer, ranging from $0.3 \mathrm{~kb}$ to $1.5 \mathrm{~kb}$. Nearly 20,000 bands were scored in the 169 analysed plants. There were clear differences in the banding patterns among accessions but no differences were detected between the control and the treated samples from the same accession, no matter which storage protocol was used (Fig. 1).

Table 1. List of primers selected for the RAPD assay, annealing temperature selected for each RAPD primer, and number of RAPD loci detected in the 51 hop accessions analysed.

\begin{tabular}{lccc}
\hline Primer & Sequence & Annealing T & Detected bands \\
\hline OPA 01 & CAGGCCCTTC & $42{ }^{\circ} \mathrm{C}$ & 12 \\
OPA 02 & TGCCGAGCTG & $42{ }^{\circ} \mathrm{C}$ & 11 \\
OPA 03 & AGTCAGCCAC & $51{ }^{\circ} \mathrm{C}$ & 10 \\
OPA 04 & AATCGGGCTG & $40{ }^{\circ} \mathrm{C}$ & 13 \\
OPA 05 & AGGGGTCTTG & $44{ }^{\circ} \mathrm{C}$ & 10 \\
OPA 06 & GGTCCCTGAC & $45^{\circ} \mathrm{C}$ & 9 \\
OPA 07 & GAAACGGGTG & $39{ }^{\circ} \mathrm{C}$ & 11 \\
OPA 08 & GTGACGTAGG & $42{ }^{\circ} \mathrm{C}$ & 13 \\
OPA 09 & GGGTAACGCC & $42{ }^{\circ} \mathrm{C}$ & 15 \\
OPA 10 & GTGATCGCAG & $40{ }^{\circ} \mathrm{C}$ & 12 \\
OPA 11 & CAATCGCCGT & $42{ }^{\circ} \mathrm{C}$ & 9 \\
Total & & & 125 \\
Mean & & & $11.36 \pm 1.85$ \\
\hline
\end{tabular}


Vol. 18 (2009): 144-151.

Table 2. List of accessions analysed using AFLP, primer combination assayed, and number of DNA fragments detected.

\begin{tabular}{|c|c|c|c|c|c|c|c|c|c|}
\hline \multirow[b]{2}{*}{ (*)Accessions } & \multicolumn{9}{|c|}{ Primer combinations } \\
\hline & agc/cat & $\mathrm{agc} / \mathrm{ctt}$ & aga/cat & aga/ctt & $\mathrm{aac} / \mathrm{ctt}$ & $\begin{array}{l}\text { acc/ } \\
\text { cat }\end{array}$ & $\begin{array}{l}\mathrm{acc} / \\
\mathrm{ctt}\end{array}$ & $\begin{array}{l}\text { Total } \\
\text { (1) }\end{array}$ & $\begin{array}{l}\text { Mean } \\
\text { (1) }\end{array}$ \\
\hline USDA 21055 Cold (2)/Cryo (2) & & & & & & & & & \\
\hline F 2n Oregon, USA -Breeding & 74 & 72 & 60 & 70 & 78 & 70 & 74 & 498 & $\begin{array}{r}71.1 \\
+5.6\end{array}$ \\
\hline USDA $62013 \times U S D A 6616-35 M$ & & & & & & & & & \\
\hline USDA 21120 Cold (2)/Cryo (2) & & & & & & & & & \\
\hline F 2n Oregon, USA -Breeding & 69 & 67 & 62 & 72 & 86 & 70 & 74 & 500 & $\begin{array}{r}71.4 \\
\pm 7.5\end{array}$ \\
\hline $19005 \times 19046 M$ & & & & & & & & & \\
\hline Calicross Cold (2)/Cryo (2) & & & & & & & & & \\
\hline F 2n New Zealand -Cultivar & 71 & 75 & 65 & 78 & 86 & 70 & 74 & 519 & $\begin{array}{l}74.1 \\
\pm 6.7\end{array}$ \\
\hline California Cluster $\times$ Fuggle seedling & & & & & & & & & \\
\hline Tardif de Bourgogne Cold (2)/Cryo (2) & & & & & & & & & \\
\hline F 2n France Cultivar & 62 & 71 & 61 & 80 & 73 & 70 & 74 & 491 & $\begin{array}{l}70.1 \\
\pm 6.7\end{array}$ \\
\hline Clonal selection landrace, Alsace & & & & & & & & & \\
\hline Missouri 3 Cryo (4) & & & & & & & & & \\
\hline F 2n Missouri, USA -Wild & 70 & 70 & 66 & 79 & 74 & 70 & 74 & 503 & $\begin{array}{r}71.8 \\
\pm 4.2\end{array}$ \\
\hline Selected from wild in Missouri & & & & & & & & & \\
\hline USDA 64033M Cold(2) & & & & & & & & & \\
\hline M 2n Oregon, USA -Breeding & 68 & 62 & 61 & 72 & 72 & 70 & 74 & 479 & $\begin{array}{l}68.4 \\
\pm 5.1\end{array}$ \\
\hline German female $7 k 491 \times O P$ & & & & & & & & & \\
\hline Colorado 3-1 Cold (2) & & & & & & & & & \\
\hline F 2n Colorado, USA -Wild & 67 & 63 & 57 & 74 & 78 & 70 & 74 & 483 & $\begin{array}{l}69 \\
\pm 7.3\end{array}$ \\
\hline Selected from wild in Colorado & & & & & & & & & \\
\hline Willamette Cold (2) & & & & & & & & & \\
\hline F 3n Oregon, USA -Cultivar & 64 & 68 & 61 & 71 & 82 & 70 & 74 & 490 & $\begin{array}{l}70 \\
\pm 6.8\end{array}$ \\
\hline USDA $21003 \times$ Fuggle & & & & & & & & & \\
\hline Vojvodina Cold (2) & & & & & & & & & \\
\hline F 2n Yugoslavia-Cultivar & 63 & 67 & 54 & 72 & 82 & 70 & 74 & 482 & $\begin{array}{l}68.8 \\
\pm 8.8\end{array}$ \\
\hline Northern Brewer $\times$ male & & & & & & & & & \\
\hline Hallertauer Magnum Cold (2) & & & & & & & & & \\
\hline F 2n Germany -Cultivar & 65 & 65 & 59 & 74 & 83 & 70 & 74 & 490 & $\begin{array}{l}70 \\
\pm 7.9\end{array}$ \\
\hline Galena $\times$ German male $75 / 5 / 3$ & & & & & & & & & \\
\hline Total (2) & 673 & 680 & 606 & 742 & 794 & 700 & 740 & 4935 & $\begin{array}{l}70.5 \\
\pm 6.1\end{array}$ \\
\hline Mean (2) & $67.3 \pm 3.8$ & $68 \pm 4.1$ & $60.6 \pm 3.5$ & $74.2 \pm 3.5$ & $79.4 \pm 5.2$ & 70 & 74 & & \\
\hline
\end{tabular}

(1) Total and mean of bands per primer combination.

(2) Total and mean of bands per accession.

(*) Information supplied: Accession name. Treatment and number of treated samples (cold, cold storage; cryo, cryopreservation). Sex: F, female; M, male. Ploidy: 2n, diploid; 3n, triploid. Origin. State: breeding (line); wild; cultivar.

Pedigree. 


\section{AGRICULTURAL AND FOOD SCIENCE}

Peredo, E.L. et al. In vitro genetic stability of hops

Table 3. Percentages of monomorphic and polymorphic MSAP loci detected in each cultivar. The polymorphic loci are arranged by behaviour category: variation present all analysed samples of both treatments, in all samples of one treatment (cryopreservation or cold storage), and present only in a single plant (no specific pattern of variation detected).

\begin{tabular}{lccccc}
\hline & Monomorphic loci & \multicolumn{4}{c}{ Polymorphic loci } \\
\cline { 2 - 6 } \cline { 4 - 6 } & & $\begin{array}{c}\text { In both } \\
\text { treatments }\end{array}$ & $\begin{array}{c}\text { Only in } \\
\text { cryopreservated }\end{array}$ & $\begin{array}{c}\text { Only cold } \\
\text { stored }\end{array}$ & Singletons \\
\hline USDA 21055 & 61.72 & 26.23 & 5.07 & 4.29 & 2.73 \\
Calicross & 63.3 & 26.59 & 2.66 & 2.66 & 4.78 \\
Tardif de Bourgogne & 52.6 & 23.7 & 9.82 & 8.67 & 5.19 \\
\hline
\end{tabular}

In the AFLP analysis, clear and specific patterns were detected for each of the 10 accessions tested. The average number of loci per sample and primer combination was $70.5 \pm 6.05$ (Table 2), ranging in size from $130 \mathrm{bp}$ to $450 \mathrm{bp}$. The maximum number of detected DNA fragments per accession and primer combination was 86 (EcoAAC/ MseCTT, 'USDA 21120' and 'Calicross') and the minimum 54 (EcoAGA/MseCAT, 'Vojvodina'). Primer combinations EcoACC/MseCAT and ACC/ CTT did not produce differing patterns among accessions. The most bands were detected in all the accessions with EcoAAC/Mse CTT (79.4 \pm 5.2$)$ while the fewest was with AGA/ACT (60.0 \pm 3.5$)$. Although nearly 19,000 bands were scored in total, no differences between the band patterns of the controls and the cryopreserved or cold stored samples were detected.
Six primer combinations were used in the MSAP analysis which produced a total of 617 clearly detected loci. Clear and repetitive peaks were detected with the automatic sequencer (Fig. 2 ). The mean number of observed loci per primer combination and cultivar was 34.94 and over 6200 bands were scored. For each accession, and in all the primer combinations tested, variations in the epigenetic profiles were detected between the treated samples and the control. The percentage of monomorphic loci detected in each accession was $61.7 \%$ ('USDA 21055'), 63.3\% ('Calicross'), and $52.6 \%$ ('Tardif de Bourgogne') (Table 3). Each polymorphic locus was assigned to one of the following categories: polymorphism present in both treatments, exclusively in the cryopreserved samples, only in cold stored samples, and singleton (change present in just one plant under the same

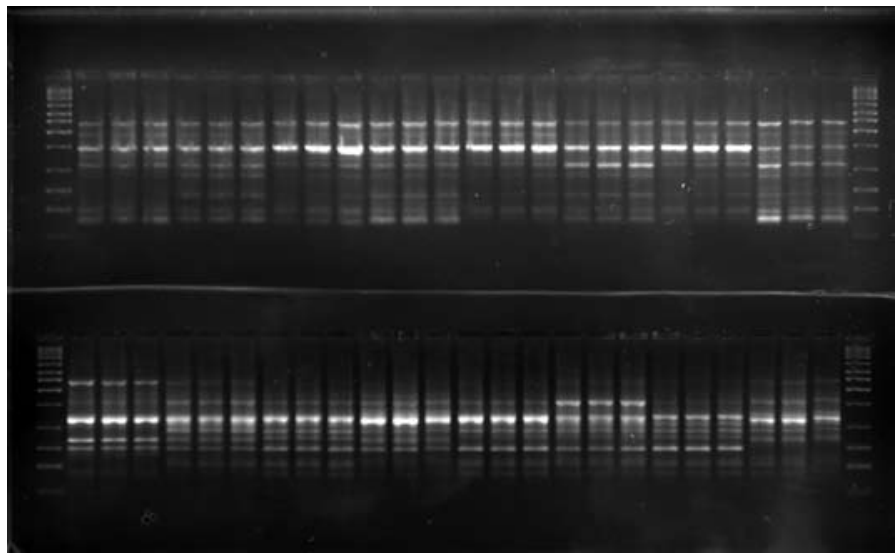

Fig. 1. Examples of RADP patterns detected in several hop accession with RAPD primer OPA 01 . For each accession no differences were found between the control and treated samples (cold stored or cryopreserved). In each group of three: control, and two treated samples. 
Vol. 18 (2009): 144-151.
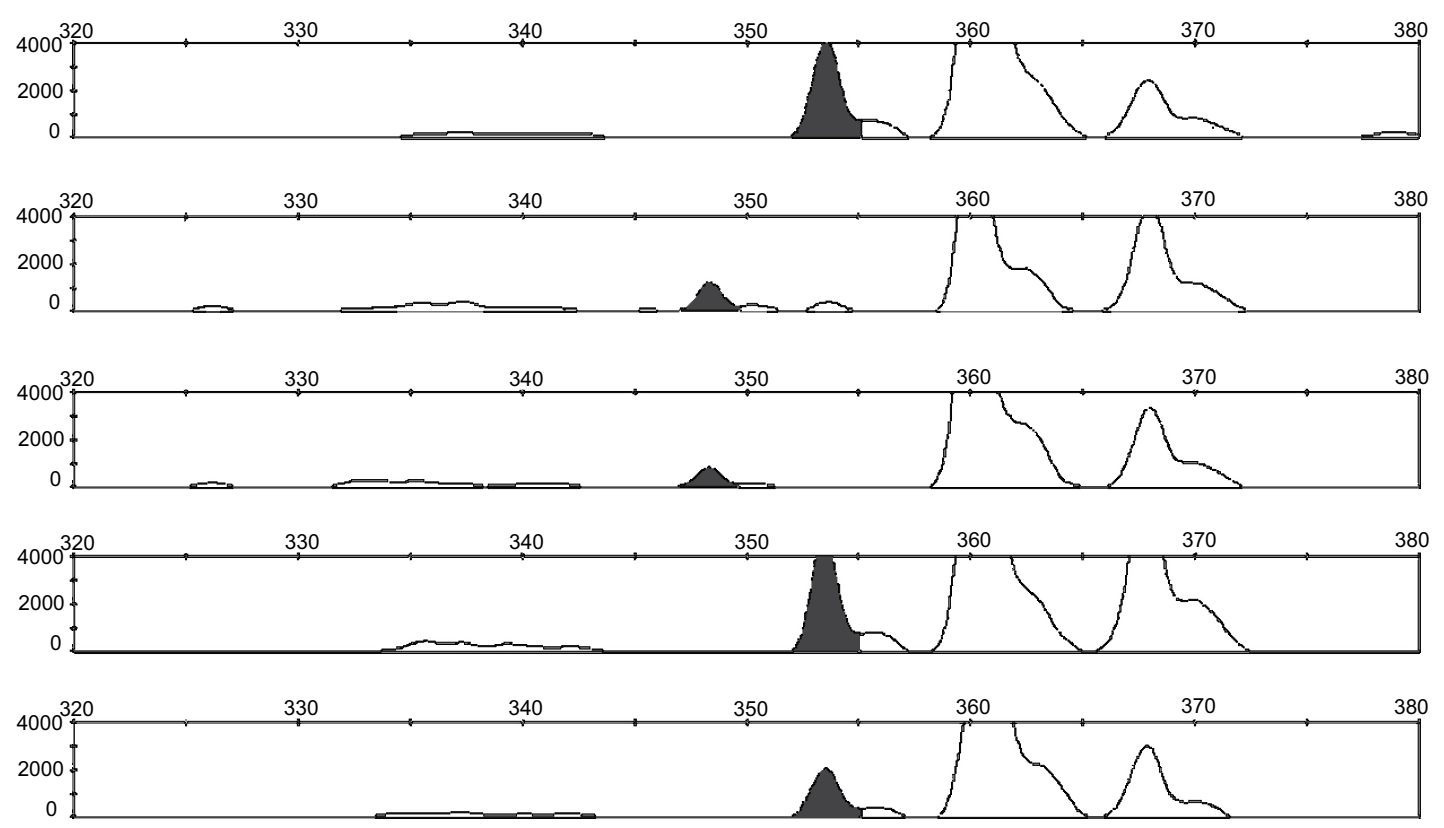

Fig. 2. Example of methylation changes detected with MSAP in accession 'Tardif de bourgone'. From top to bottom: control plant, two cryopreseved plants, two cold stored plants.

treatment). Most of the detected variation was shared by both treatments, ranging from $23.7 \%$ to $26.7 \%$ depending on the accession. The amount of variation that could be specifically related to either of the treatments was relatively low ranging from 2.6 to $9.8 \%$ of the total detected MSAP loci for the cryopreserved plants, and from 2.6 to $8.6 \%$ in cold-stored plants. The variation that could not be attributed to either or both of the treatments ranged from 2.7 to $5.19 \%$.

\section{Discussion}

Cryopreservation and cold storage are two of the most appropriate techniques to conserve large collections of plants (Ashmore 1997). Both techniques involve in vitro culture manipulation of the stored tissues, that could be considered a potential risk for the generation of genetic instability (Brar and Jain 1998). An increasing number of studies indicate that plants recovered from cold storage or slow growth have no genetic alterations (Hao et al. 2004, Renau-Morata et al. 2006). This is also the case observed for these cold-stored hops; no RAPD or AFLP variation could be detected in any of analysed accessions. Similar data were obtained for cryopreserved hop accessions. No changes attributed to somaclonal variation were detected in the eleven accessions subjected to cryopreservation when analysed either with RAPD or AFLP. Based on these observations, it is reasonable to deduce that both cold storage and cryopreservation can be used for the routine storage of hops. Similar results are reported for other species in the current literature: no somaclonal variation was observed in cryopreserved apple (Hao et al. 2001, Lui et al. 2004), or grape and kiwi (Zhaj et al. 2003). However, it is important to note that somaclonal variation was reported in Dendrathema grandiflora (Martín and Gonzalez-Benito 2005), and Hypericum perforatum (Urbanová et al. 2006)

Epigenetic changes are a common cause for somaclonal variation, due to the stresses generated 
Peredo, E.L. et al. In vitro genetic stability of hops

during in vitro culture, and these changes could possibly generate altered phenotypes in the recovered plants. In our study, clear epigenetic changes were detected in each accession when compared to the potted plants used as controls. Over $26 \%$ of the detected MSAP loci shared some sort of modification after cold storage or cryopreservation. In any of the cultivars, the variation explained by the storage method itself was higher than the amount of variation shared by both treatments. This might be explained by the epigenetic changes related to physiological alterations produced by in vitro establishment. DNA methylation is a dynamic mechanism by means of which plasticity is induced by environmental and/or ontogenic signals (Ramchandani et al. 1999). Therefore, it is not surprising that there is a correlation between the physiological changes produced due to in vitro growth and epigenetic alterations detected in all the in vitro plants. Similar results were found in previous studies of in vitro establishment in hop in which nearly $30 \%$ of the detected MSAP loci were polymorphic (Peredo et al. 2009). However, as there are exclusive methylation changes in the cold-stored and cryopreserved plants, we can assume that each protocol is an additional source of epigenetic variation. Methylation changes were also reported in cryopreserved apple and strawberries (Hao et al. 2001 and 2002) and citrus callus under slow growth (Hao et al. 2004). Few studies on the genetic stability of hop plants are available. Patzak (2003) described an increased frequency of genetic changes after thermotherapy of in vitro hop meristems. MSAP polymorphisms were detected in hop plants regenerated from callus (Peredo et al. 2006).

In summary, methylation changes were detected in both cold stored and cryopreserved plants, although no genetic changes were identified using RAPD and AFLP. We can assume that the genotypes behave similarly when cryopreserved or cold stored as no major differences were found in a range of hop accessions. Both protocols are suitable for use as standard storage methods, but it is important to take into account that a considerable amount of epigenetic change can be induced, particularly during the in vitro process.
Acknowledgments:

This research was supported by funds from the Spanish Ministry of Science and Technology and FEDER (project MCT-02-AGL-02472). E.L.P. is in receipt of an F.P.U. grant. All the plant material was provided by the USDA-ARS National Clonal Germplasm Repository (Corvallis, OR, USA) and supported by CRIS 5358-21000-033-00D.

\section{References}

Ashmore S.E. 1997. Status report on the development and application of in vitro techniques for the conservation and use of plant genetic resources. International Plant Genetic Resources Institute. Rome, Italy.

Brar D.S. \& Jain S.M. 1998. Somaclonal variation: Mechanism and applications in crop improvement. In: S.M. Jain, D.S. Brar, B.S. Ahloowalia (eds.) Somaclonal variation and induced mutations in crop improvement. Kluwer Academic Press, Dordrecht, Boston, London. pp. 15-24.

Cervera M.T., Cabezas J.A., Sancha J.C., Martínez de Toda F. \& Martínez-Zapater J.M. 1998. Application of AFLPs to the characterization of grapevine Vitis vinifera L. genetic resources. A case study with accessions from Rioja, Spain. Theorical and Applied Genetics 97: 51-59.

Cervera M.T., Ruiz-García L. \& Martínez-Zapater J.M. 2002. Analysis of DNA methylation in Arabidopsis thaliana based on methylation-sensitive AFLP markers. Molecular Genetics and Genomics 268: 543-552.

Finnegan E.J., Genger R.K., Peacock W.J. \& Dennis E.S. 1998. DNA methylation in plants. Annual Review of Plant Physiology and Plant Molecular Biology 49: 223-247.

Harding K. 2004. Genetic integrity of cryopreserved plant cells: A review. CryoLetters 25: 3-22.

Hao Y.J., Liu Q.L. \& Deng X.X. 2001. Effect of cryopreservation on apple genetic resources at morphological, chromosomal and molecular levels. Cryobiology 43: 46-51.

Hao Y.J., You C.X. \& Deng X.X. 2002. Analysis of ploidy and the patterns of amplified fragment length polymorphism and methylation sensitive amplified polymorphism in strawberry plants recovered from cryopreservation. CryoLetters 23: 37-46.

Hao Y.J., Wen X.P. \& Deng X.X. 2004. Genetic and epigenetic evaluations of citrus calluses recovered from slow-growth culture. Journal of Plant Physiology 161: 479-484.

Lui Y., Wang X. \& Liu L. 2004. Analysis of genetic variation in surviving apple shoots following cryopreservation by vitrification. Plant Science 166: 677-685.

Martín C. \& González-Benito E. 2005. Survival and genetic stability if Dendrathema grandiflora Tzvelev shoot after cryopreservation by vitrification and encapsulationdehydration. Cryobiology 51: 281-289.

Moir M. 2000. Hops - A millennium review. Journal of the American Society of Brewing Chemists 58: 131-146.

Patzak J. 2003. Assessment of somaclonal variability in hop (Humulus lupulus L.) in vitro meristem cultures and 


\section{AGRICULTURAL AND FOOD SCIENCE}

\section{Vol. 18 (2009): 144-151.}

clones by molecular methods. Euphytica 131: $343-$ 350.

Peredo E.L., Revilla M.A. \& García-Arroyo R. 2006. Assessment of genetic and epigenetic variation in hop plants regenerated from sequential subcultures. Journal of Plant Physiology 163: 1071-1079.

Peredo E.L., García-Arroyo R. \& Revilla M.A. 2009. Epigenetic changes detected in micropropagated hop plants, Journal of Plant Physiology. Available on the Internet: Doi:10.1016/j.jplph. 2008.12.015.

Pillay M. \& Kenny S.T. 1996. Random amplified polymorphic DNA (RAPD) markers in hop, Humulus lupulus: level of genetic variability and segregation in $\mathrm{F}_{1}$ progeny. Theorical and Applied Genetics 92: 334-339.

Ramchandani S., Bhattacharya S., Cervoni N. \& Szyf M. 1999. DNA methylation is a reversible biological signal. Proceedings of the National Academy of Sciences of the United States of America 96: 6107-6112.

Reed B.M., Okut N., D’Achino J., Narver L. \& DeNoma J. 2003 Cold Storage and cryopreservation of hops $(\mathrm{Hu}-$ mulus lupulus) shoot cultures through applications of standard protocols. CryoLetters 24: 389-396.
Renau-Morata B., Arrillaga I. \& Segura J. 2006. In vitro storage of cedar shoot culture under minimal growth conditions. Plant Cell Reports 25: 636-642.

Urbanová M., Kosuth J. \& Cellárová E. 2006 Genetic and biochemical analysis of Hypericum perforatum L. plants regenerated after cryopreservation. Plant Cell Reports 25: $140-147$.

Williams J.G.K., Kubelik A.R., Livak K.J., Rafalski J.A. \& Scott V.T. 1990. DNA polymorphisms amplified by arbitrary primers are useful as genetic markers. Nucleic Acids Research 18: 6531-6535.

Vos P., Hogers R., Bleeker M., Reijans M., Van de Lee T. \& Hornes M. 1995. AFLP: a new technique for DNA fingerprinting. Nucleic Acids Research 23: 4407-4414.

Viterbo A., Altman H.D. \& Rabinowitch A. 1994. In vitro propagation and germplasm cold-storage of fertile and male-sterile Allium trifoliatum subsp. hirsutum. Genetic Resources and Crop Evolution 41: 87-98.

Zhaj Z., Wu Y., Engelmann F., Chen R. \& Zhao Y. 2003. Genetic stability assessments of plantlets regenerated from cryopreserved in vitro cultured grape and kiwi shoot-tips using RAPD. CryoLetters 24: 315-322. 